\title{
Energy Efficient Scheme for Cognitive Radios Utilizing Soft Sensing
}

\author{
AbdulRahman Alabbasi, Zouheir Rezki , Basem Shihada \\ CEMSE Division, King Abdullah University of Science and Technology \\ Email: \{abdulrahman.alabbasi, zouheir.rezki, basem.shihada\}@kaust.edu.sa
}

\begin{abstract}
In this paper we propose an energy efficient cognitive radio system. Our design considers an underlaying resource allocation combined with soft sensing information to achieve a sub-optimum energy efficient system. The sub-optimality is achieved by optimizing over a channel inversion power policy instead of considering a water-filling power policy. We consider an Energy per Goodbit (EPG) metric to express the energy efficient objective function of the system and as an evaluation metric to our system performance. Since our optimization problem is not a known convex problem, we prove its convexity to guarantee its feasibility. We evaluate the proposed scheme comparing to a benchmark system through both analytical and numerical results.

Index Terms-Spectrum sharing, Energy efficiency, Spectrum sensing, Resource allocation,
\end{abstract}

\section{INTRODUCTION}

The carbon footprint is a popular measure of "greenness". However, from the engineering perspective in wireless systems, it is hard to translate this criteria directly to quantifiable parameters. The notion of "green" technology in wireless systems can be made meaningful with a comprehensive evaluation of energy savings and performance in a practical system. The definition of adequate metrics is still an open problem and standards organizations are making efforts to define energy efficiency metrics for wireless networks. Making communication devices and spectrum usage greener will not only benefit the environment, but also increase the profitability of business for communication companies. In addition, green spectrum reduces the dependency on the legacy fuel supplies and lead to sustainable prosperity. One of the proposed green communication applications is cognitive radio (CR) system [1]. The combination of cognition system and energy efficiency technologies can be done in several ways as shown in [2], [3], [4].

Cognitive Radio (CR) has been proposed to overcome the inefficient use of frequency spectrum and its resulting scarcity [5]. In $\mathrm{CR}$, secondary users (SU) may share the bands of the primary users (PU) through different schemes. For instance, opportunistic sharing and underlaying sharing are two schemes that have been investigated in the literature [5]. The formal technique forces the SU to sense the PUs spectrum holes (unused PU bands) and transmit only on these bands. On the other hand, underlaying sharing allows SU to transmit on PUs bands with a certain interference threshold [5]. Combining both techniques results in an improved performance compared to the conventional sharing [6]. Some papers consider PU transmitter detection with soft sensing decision instead of hard decision. Soft (hard) sensing decision implies that we are utilizing the sensing information into the scheme befogre (after) deciding the existence of PU. In CR environment it is more realistic to consider that SU does not have a prior knowledge of the PU's signal and its Channel State Information (CSI). We take this into consideration in our system and sensor design.

Researchers have been considering optimal energy efficient systems by minimizing the system energy while preserving its Quality of Service (QoS) parameters, such as rate, delay, etc. [7]. However, it has been indicated in [8] that optimizing the Energy Per Goodbits (EPG) yields to an improvemed result in terms of energy efficiency.

In this paper we propose and analyse an energy efficient communication system with spectrum sharing utilizing sensing information about PU, using an adaptive power and resource allocation scheme. In our analysis we consider the case where the PU is assumed to be active all the time. Our framework is divided into proposed and benchmark systems. In the proposed system we assume the existence of an on-board sensor at the SU, while the benchmark system depend only on the fact that PU is active and has to be protected with the same interference level all the time. The proposed system (of SU) uses its on-board sensor, frequently, to estimate and have a real time information about the PU activity to aggregate on the secondary to primary channel as much as possible, within the tolerable interference threshold.

Our proposed system has unique contributions as follows. We consider EPG metric with sensing information to evaluate the system performance, where the authors in [6], [10] considered only the capacity metric. Additionally, we proved the convexity of the proposed formulation. Analytical results are provided for both the proposed and benchmark systems.

This paper is organized as follows. Section II describes our system model with some related background on PU sensing approach. Section III discusses the problem formulation of both the benchmark and proposed systems. Followed by Section IV, which specifies the general derived analytical results for specific distributions. Finally, some numerical results are presented in Section V.

\section{System Model AND Related BACKGROUND}

\section{A. System Model}

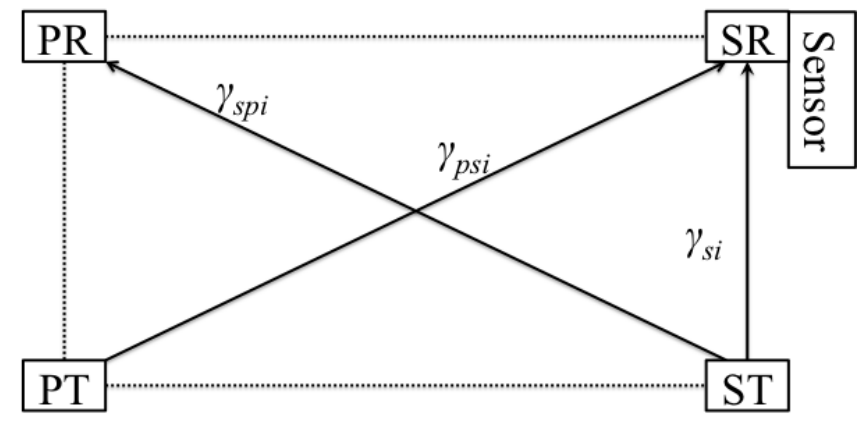

Fig. 1. System Model.

Our system model seeks to achieve energy efficient system through a sub-optimal CR resource allocation scheme. We consider a spectrum sharing protocol where the $\mathrm{SU}$ is sharing the available spectrum with the PU. Figure 1 shows our system model for a sub-carrier index $i$, whereas $i \in\{1, \ldots, N\}$. In Figure 1 the fading channels between 
primary transmitter (PT) and secondary receiver (SR), primary receiver (PR) and secondary transmitter (ST), and ST and SR are designated by $h_{p s i}, h_{s p i}, h_{s i}$, and their corresponding absolute squares $\gamma_{p s i}, \gamma_{s p i}, \gamma_{s i}$, respectively. The previously mentioned channels gains are assumed to be independent.

It is assumed that the sensor is on the SR side. Additionally, we consider that both ST and SR share the knowledge about the sensing information $\left(\zeta_{i}\right), \gamma_{s i}$ and the average value of $\gamma_{s p i}\left(\overline{\gamma_{s p i}}\right)$ through a feedback channel. It is assumed that PT transmits on maximum power $\left(P_{p}\right)$ all the working times. On the other hand, ST has an adaptive power allocation policy (to be described later) denoted as $P_{s i}$, differs per each sub-carrier $i$. An absolute condition on our system model is that the interference toward the PR to be limited within a certain interference threshold.

\section{B. Sensing Background}

In this section we provide the necessary background for incorporating the sensing information in our proposed system model. The linkage between the problem formulation and sensing information is done through the SU-to-PU average interference constraint, at which we include the sensing data. In addition to the original averaging of SU-to-PU interference, which is done over $\gamma_{s i}$ and $\gamma_{s p i}$, we also average over sensing data received by the SU sensor (detector). In our framework the sensor senses the channels for $T_{s}$ then start transmitting for a period of time $T_{c}$. Where we assume that $T_{c}>T_{s}$, such that $T_{s}$ does not affect our performance. Additionally, we assume that the channel coherence time is large enough so that it does not change within two periods of sensing.

Since we do not have a prior knowledge about PU signal or channel, we use energy detection. As known in the literature, the output of the energy detector is [11],

$$
\zeta_{i}\left(N_{s}\right)=\frac{1}{N_{s}} \sum_{n=1}^{N_{s}}\left|y_{i}(n)\right|^{2},
$$

where $N_{s}$ is the number of sensing samples, and $y_{i}(n)$ is the received signal at SR sensor defined as,

$$
y_{i}(n)=\left\{\begin{array}{lll}
\eta_{i}(n) & \mathcal{H}_{0}: & \text { (PU is idle) } \\
h_{p s i}(n) x_{i}(n)+\eta_{i}(n) & \mathcal{H}_{1}: & \text { (PU is active) }
\end{array} .\right.
$$

where, $x_{i}(n)$ is the PU data, and $\eta_{i}(n)$ is the additive white Gaussian noise (AWGN), with zero mean and unity variance, at the sensor side.

The authors of [11] have done a thorough investigation on the probability density function (PDF) of $\zeta_{i}\left(N_{s}\right)$ for both cases $\mathcal{H}_{0}$ and $\mathcal{H}_{1}$. Following the same general approach, we obtain the PDF of both $\mathcal{H}_{0}$ and $\mathcal{H}_{1}$ as $g_{0}\left(\zeta_{i}\right)$ and $g_{1}\left(\zeta_{i}\right)$, and their corresponding means $\mu_{0}$, $\mu_{1}$, and variances $\delta_{0}^{2}, \delta_{1}^{2}$, respectively. Deciding whether PU exists or not requires an optimal decision mechanism. We use the likelihood function of the PDFs for the two hypotheses as follows,

$$
\frac{g_{0}\left(\zeta_{i}\right)}{g_{1}\left(\zeta_{i}\right)} \geq \gamma_{u i}
$$

where $\gamma_{u i}$ is the threshold which identifies that PU is idle if (3) is satisfied.

By solving the inequality in (3), we obtain a set of $\zeta_{i}$ that lies within a region $Z_{0}$ and satisfies (3), meaning that PU is idle. On the other hand, if (3) is not satisfied, then the set of the resulting values of $\zeta_{i}$ lie within a region $Z_{1}$, where $Z_{1}=Z_{0}{ }^{c}=1-Z_{0}$, and the PU is declared to be active, so we refrain from transmitting.

\section{PROBLEM Formulation}

In this section, we begin by discussing the benchmark system with some related analysis in Section III-A, then the proposed system formulation and its analysis is presented in Section III-B.

\section{A. Benchmark System Formulation}

In this sub-section we discuss the problem formulation of the benchmark system which is used as reference to evaluate the proposed system. Our problem is to minimize the objective function, which is the EPG metric, subject to several constraints as follows, this problem is called $P_{0}$,

$$
\begin{aligned}
& P_{0}: \quad \min _{P_{s i}(\gamma)} \frac{\sum_{i=1}^{N} P_{s i}(\gamma)}{\sum_{i=1}^{N} \log \left(1+\frac{P_{s i}(\gamma) \gamma_{s i}}{1+P_{p} \gamma_{p s i}}\right)} \\
& \text { s.t. } \quad P_{s i}(\gamma) \leq P_{\max } \\
& \sum_{i=1}^{N} \log \left(1+\frac{P_{s i}(\gamma) \gamma_{s i}}{1+P_{p} \gamma_{p s i}}\right) \geq R_{\text {min }} \\
& E_{\gamma}\left[P_{s i}(\gamma) \gamma_{p i}\right] \leq P_{a v e}
\end{aligned}
$$

where, $P_{s i}(\gamma)$ and $P_{p}$ are the transmission powers of SU and PU respectively, at sub-carrier $i, N$ is the total number of subcarriers. For the benchmark system the variable appears in (4d) is equivalent to both $\gamma_{s i}$ and $\gamma_{s p i}$. The parameters $P_{\max }$ and $R_{\min }$ are assigned constants for maximum instantaneous power per subcarrier of SU and minimum acceptable rate of SU. $P_{\text {ave }}$ is the average power constraint on the received interference from ST at PR. In $P_{0}$ (4), the objective function is described in (4a) as the instantaneous EPG, where the constraints are described as bellow. First, the maximum instantaneous power constraint over $N$ communication sub-carriers, as in (4b). Second, the minimum instantaneous SU rate, as in (4c). Finally, the average SU to PU interference constraint, as in (4d), where in the benchmark system we average over only $\gamma_{s i}$ and $\gamma_{s p i}$.

It is clear that our framework considers only one average constraint while the other constraints and the objective function are instantaneous ones. A practical implementation for this framework is when $\mathrm{SU}$ is operating on a real time, delay intolerance, communication system. While, on the other hand, the PU is operating on a delay tolerance system.

We start solving the average constraint by considering, without loss of generality, the $\mathrm{PU}$ is active with probability $\operatorname{Pr}\{\mathrm{PU}$ is $\mathrm{ON}\}$ and $\mathrm{PU}$ is idle with probability $\operatorname{Pr}\{\mathrm{PU}$ is $\mathrm{OFF}\}$. Then by conditional expectation, (4d) is expanded into,

$$
\begin{array}{r}
E_{\gamma}\left[P_{s i}(\gamma)\right]=\operatorname{Pr}\{\mathrm{PU} \text { is } \mathrm{ON}\} E_{\gamma}\left[P_{s i}(\gamma) \gamma_{s p i} \mid \mathrm{PU} \text { is } \mathrm{ON}\right] \\
+\operatorname{Pr}[\mathrm{PU} \text { is } \mathrm{OFF}] E_{\gamma}\left[P_{s i}(\gamma) \mid \mathrm{PU} \text { is } \mathrm{OFF}\right] \leq P_{\text {ave }},
\end{array}
$$

where,

$$
P_{\text {ave }}=\left\{\begin{array}{ll}
P, & \mathcal{H}_{0}:(\mathrm{PU} \text { is } \mathrm{OFF}) \\
Q_{\text {int }} & \mathcal{H}_{1}:(\mathrm{PU} \text { is } \mathrm{ON})
\end{array} .\right.
$$

where, $Q_{\text {int }}$ and $P$ are the average power constraint on ST when the PU is active and idle, respectively. $\operatorname{Pr}\{$.$\} is the probability of the$ in-arguement event. Since we assume that PU is active all the time, then $\operatorname{Pr}\left\{\mathcal{H}_{1}\right\} \rightarrow 1$. Therefore, (5) is reduced to,

$$
E_{\gamma}\left\{P_{s i}(\gamma) \gamma_{s p i}\right\} \leq Q_{\text {int }}
$$

To solve (7) we choose a power policy that is similar to the channel inversion power policy. The chosen power policy is depicted as follows,

$$
P_{s i}=\left\{\begin{array}{ll}
0, & \gamma_{s i}<\gamma_{v i} \\
\frac{\sigma_{i}}{\gamma_{s i}}, & \gamma_{s i} \geq \gamma_{v i}
\end{array} .\right.
$$


where $\gamma_{v i}$ is the SUs channel threshold to be optimized, which gives an indication about the SU channel $\gamma_{s i}$ (whether it is in deep fading, so we stop transmission, or in good condition, so we start transmission). The reason of choosing channel inversion power policy in (8) is that the same power allocation have been assumed in the proposed system case. Therefore, this keeps a fair comparison between both proposed and benchmark system.

Knowing that $\gamma$ consists of both $\gamma_{s i}$ and $\gamma_{s p i}$, which are independent random variables, we begin solving the expectation in (7). It is observed that even though we have a feedback channel between SR and ST to share the channel information, we consider the $\gamma_{s i}$ as a random variable in order to obtain the optimal value of $\gamma_{v i}$. This does not contradict with the fact that we know $\gamma_{s i}$ since we need to find $\gamma_{v i}$ to optimally allocate the power policy in (8). Taking into account the assumed power policy in (8), then we can separate the expectation of (7) into two integrals as follows,

$$
\sigma_{i} \int_{\gamma_{v i}}^{\infty} \frac{1}{\gamma_{s i}} f_{\gamma_{s i}}\left(\gamma_{s i}\right) d \gamma_{s i} \int_{0}^{\infty} \gamma_{s p i} f_{\gamma_{s p i}}\left(\gamma_{s p i}\right) d \gamma_{s p i} \leq Q_{i n t}
$$

The first integral results in a general function of $\gamma_{v i}, G\left(\gamma_{v i}\right)=$ $\int_{\gamma_{v i}}^{\infty} \frac{1}{\gamma_{s i}} f_{\gamma_{s i}}\left(\gamma_{s i}\right) d \gamma_{s i}$, which depends on the PDF of $\gamma_{s i}$ The second integral results in $\overline{\gamma_{s p i}}$, the average value of the $\gamma_{s p i}$ channel. Therefore, (9) becomes

$$
\sigma_{i} G\left(\gamma_{v i}\right) \leq Q_{i n t}^{\prime}
$$

where $Q_{i n t}^{\prime}=\frac{Q_{i n t}}{\gamma_{s p i}}$.

To show the analytical solution of the $P_{0}$ we transform the objective function (4a) into a convex form. It is proven in [8] that the minimization of (4a) is equivalent to minimizing the following function,

$$
g\left(P_{s i}, t\right)=\sum_{i=1}^{N} P_{s i}-t\left(\sum_{i=1}^{N} \log \left(1+\frac{P_{s i} \gamma_{s i}}{1+P_{p} \gamma_{s i}}\right)\right) .
$$

with respect to $t$ and $P_{s i}$.

The Lagrangian function of problem $P_{1}$ [12] is constructed while including (8)'s inequality as part of the Lagrangian function and we leave the maximum power constraint inequality (4b) to a later step (just like in the case of iterative water-filling approach). Finally, the Lagrangian function is expressed as follows,

$$
\begin{aligned}
L= & \\
& \sum_{i=1}^{N} \frac{\sigma_{i}}{\gamma_{s i}}-t \sum_{i=1}^{N} \log \left(1+\frac{\sigma_{i}}{1+P_{p} \gamma_{p s i}}\right) \\
& -\lambda_{1}\left[\sum_{i=1}^{N} \log \left(1+\frac{\sigma_{i}}{1+P_{p} \gamma_{p s i}}\right)-R_{\min }\right] \\
& +\sum_{i=1}^{N} \lambda_{2 i}\left[\sigma_{i} G\left(\gamma_{v i}\right)-Q_{i n t}^{\prime}\right] \\
& +\sum_{i=1}^{N} \lambda_{3 i}\left[\gamma_{v i}-\gamma_{s i}\right]
\end{aligned}
$$

where $\lambda_{1}$ is the Lagrangian multiplier corresponds to (4c), $\lambda_{2 i}$ is the Lagrangian multiplier corresponds to (10), and $\lambda_{3 i}$ is the Lagrangian multiplier corresponds to inequality condition of (8).

Differentiating the Lagrangian function with respect to $\sigma_{i}$, and utilizing the Complementary Slackness conditions for constraint (10), we have two analytical solutions for $\sigma_{i}$

$$
\begin{aligned}
& \text { - } \lambda_{2 i}=0, \text { then we obtain } \frac{\sigma_{i}^{*}}{\gamma_{s i}}= \\
& \min \left(\left[t+\lambda_{1}-\frac{1+P_{p} \gamma_{p s i}}{\gamma_{s i}}\right]^{+}, P_{\max }\right)
\end{aligned}
$$

- $\lambda_{2 i} \neq 0$, then we obtain $\sigma_{i}$ by solving the following quadratic equation

$$
\sigma_{i}^{*}=\min \left(\left[\frac{-b \pm \sqrt{\left(b^{2}-4 a c\right)}}{2 a}\right]^{+}, \sigma_{m n s}\right) .
$$

where, $a=\frac{1}{\gamma_{s i}}, b=\left[\frac{1+\gamma_{p s i} P_{p}}{\gamma_{s i}}+\lambda_{2 i} Q_{i n t}^{\prime}-\left(\lambda_{1}+t\right)\right]$, and, $c=\left[\lambda_{2 i} Q_{\text {int }}^{\prime}\left(1+\gamma_{p s i} P_{p}\right)\right], \sigma_{m n s}=\operatorname{Min}\left(\sigma_{n s}^{+}, P_{\max } \gamma_{s i}\right)$, and, $\sigma_{n s}^{+}=\frac{Q_{i n t}^{\prime}}{G\left(\gamma_{v i}\right)}$.

In a similar way, differentiating with respect to $\gamma_{v i}$ leads to an analytical expression for $\gamma_{v i}^{*}$ as follows,

$$
\frac{\partial}{\partial \gamma_{v i}} G\left(\gamma_{v i}\right)=-\frac{\lambda_{3 i}}{\lambda_{2 i} \sigma_{i}}
$$

Finally, We solve the Lagrangian multipliers $\lambda_{1}, \lambda_{2 i}, \lambda_{3 i}$ through the solution of the dual problem, and $t$ can be found in a similar way to [8].

\section{B. Proposed System Formulation}

In this Section we formulate the proposed system problem and express the analytical solution of the optimal allocated power. The new formulation follows exactly the formulation of problem $P_{0}$ with some changes in (4d). Where we follow the same approach implemented to derive (7) for the benchmark case. However, in the propose system case, (7) is rewritten as,

$$
E_{\gamma_{s i}, \gamma_{s p i}, \zeta_{i}}\left\{P_{s i}\left(\gamma_{s i}, \gamma_{s p i}, \zeta_{i}\right) \gamma_{s p i}\right\} \leq Q_{i n t},
$$

where, $\gamma$ in (7) is replaced by three variables, namely, $\gamma_{s i}, \gamma_{s p i}$, and $\zeta_{i}$ (instead of two as in the benchmark system). The new problem after replacing (7) by (15) is called $P_{1}$.

Our optimization variables of $P_{1}$ are the SU transmission power $P_{s i}\left(\gamma_{s i}, \gamma_{s p i}, \zeta_{i}\right)$, sensing metric threshold $\gamma_{u i}$, and SU channel threshold $\gamma_{v i}$. Since $P_{s i}\left(\gamma_{s i}, \gamma_{s p i}, \zeta_{i}\right)$ is a function of three random variables, $\gamma_{s i}, \gamma_{s p i}$, and $\zeta_{i}$, and the expectation is a function of $P_{s i}\left(\gamma_{s i}, \gamma_{s p i}, \zeta_{i}\right)$, then we have to deal with a functional of functions. Therefore, for simplicity in solving inequality (15), we propose the channel inversion power policy as follows

$$
P_{s i}=\left\{\begin{array}{ll}
0, & \frac{g_{0}\left(\zeta_{i}\right)}{g_{1}\left(\zeta_{i}\right)}<\gamma_{u i} \quad \text { or } \quad \gamma_{s i}<\gamma_{v i} \\
\frac{\sigma_{i}}{\gamma_{s i}}, & \frac{g_{0}\left(\zeta_{i}\right)}{g_{1}\left(\zeta_{i}\right)} \geq \gamma_{u i} \quad \text { and } \quad \gamma_{s i} \geq \gamma_{v i}
\end{array} .\right.
$$

We utilize the power policy assumption in (16) and the independency among $\gamma_{s i}, \gamma_{s p i}$, and $\zeta_{i}$ to start solving the expectation in (15). It follows that (15) is expressed as,

$$
\begin{array}{r}
\sigma_{i} \int_{\gamma_{v i}}^{\infty} \frac{1}{\gamma_{s i}} f_{\gamma_{s i}}\left(\gamma_{s i}\right) d \gamma_{s i} \int_{0}^{\infty} \gamma_{s p i} f_{\gamma_{s p i}}\left(\gamma_{s p i}\right) d \gamma_{s p i} \\
\int_{Z_{0}} g_{1}\left(\zeta_{i}\right) d \zeta_{i} \leq Q_{i n t}
\end{array}
$$

where, $Z_{0}$ is the region of $\zeta_{i}$ which satisfies (3). The first and second integral can be solved similarly to the ones in (9). The third integral depends on $g_{1}\left(\zeta_{i}\right)$ the distribution of $\zeta_{i}$. Let $\int_{Z_{0}} g_{1}\left(\zeta_{i}\right) d \zeta_{i}=$ $K_{01}\left(\gamma_{u i}\right)$. Finally, (17) is reduced to,

$$
\sigma_{i} G\left(\gamma_{v i}\right) K_{01}\left(\gamma_{u i}\right) \leq Q_{i n t}^{\prime},
$$

In order to guarantee that we always achieve a feasible, optimal, solution for $P_{1}$, we prove its convexity in Appendix A.

The analytical solution of $\sigma_{i}$ is obtained by differentiating the Lagrangian function of $P_{1}$ with respect to $\sigma_{i}$, in a similar way to 
the derivation of the benchmark solution. Then $\sigma_{i}$ is expressed as,

$$
\begin{aligned}
& \frac{\sigma_{i}^{*}}{\gamma_{s i}}=\min \left(\left[\frac{t+\lambda_{1}}{1+\lambda_{2 i} G\left(\gamma_{v i}\right) K_{01}\left(\gamma_{u i}\right)}\right.\right. \\
& \left.\left.-\frac{\left(1+P_{p} \gamma_{p s i}\right)}{\gamma_{s i}}\right]^{+}, P_{+}^{w s}\right) .
\end{aligned}
$$

where

$$
P_{+}^{w s}=\min \left(\frac{\sigma_{m w s}}{\gamma_{s i}}, P_{\max }\right)
$$

and

$$
\sigma_{m w s}=\frac{Q_{i n t}^{\prime}}{G\left(\gamma_{v i}\right) K_{01}\left(\gamma_{u i}\right)}
$$

We notice that (19) takes the form of water-filling, and (20) is the clipping policy for the power allocation (19). Where the effect of sensing appears in first term of the minimum function of (19). It is proved in Appendix A that $K_{01}\left(\gamma_{u i}\right)$ is a monodically decreasing function of $\gamma_{u i}$, therefore, the larger the threshold $\gamma_{u i}$ the larger the allocated power. However to guarantee a high detection probability (low miss-detection probability) of our sensor, it is necessary to add an upper bound on $\gamma_{u i}$. Therefore, $\gamma_{u i}^{*}$ is obtained as,

$$
\gamma_{u i}^{*}=\min \left(\gamma_{u i}^{(m)}, \gamma_{u i}^{(o)}\right)
$$

where, $\gamma_{u i}^{(m)}$ is the upper bound value of $\gamma_{u i}$, and $\gamma_{u i}^{(o)}$ is the optimal value of $\gamma_{u i}$ obtained by differentiating (18) with respect to $\gamma_{u i}$ and finding it zeros (numerically). Finally, $\gamma_{v i}^{*}$ is found in a similar way to (14) while adding the effect of sensing metric.

\section{Application on Specific Distributions}

We assume that the fading channels $\gamma_{s i}, \gamma_{s p i}$, and $\gamma_{p s i}$ are independent identically distributed random variables that follow an exponential distribution. Furthermore, although $\zeta_{i}$ is not Gaussian, we use the central limit theorem to approximate it as Gaussian distribution which simplifies the analysis without loss of generality. This approximation is done for both hypotheses $\mathcal{H}_{0}$ and $\mathcal{H}_{1}$, with means $\mu_{0}, \mu_{1}$, and variances $\delta_{0}^{2}, \delta_{1}^{2}$, respectively. Since we obtain a sufficiently large number of samples for $\zeta_{i}$, it is reasonable to use the Gaussian approximation in our analysis. The corresponding PDF of both hypotheses are $g_{0}\left(\zeta_{i}\right)$ and $g_{1}\left(\zeta_{i}\right)$, and expressed as,

$$
\begin{aligned}
& g_{0}\left(\zeta_{i}\right)=\frac{\exp \left(-\frac{\left(\zeta_{i}-\mu_{0}\right)^{2}}{2 \delta_{0}^{2}}\right)}{\delta_{0}} \\
& g_{1}\left(\zeta_{i}\right)=\frac{\exp \left(-\frac{\left(\zeta_{i}-\mu_{1}\right)^{2}}{2 \delta_{1}^{2}}\right)}{\delta_{1}}
\end{aligned}
$$

Following the same assumption on $\zeta_{i}$ 's PDF, we can find that the region $Z_{0} \in\left[\rho_{1}\left(\gamma_{u i}\right), \rho_{2}\left(\gamma_{u i}\right)\right]$, which can be found as,

$$
\begin{aligned}
& \rho_{1}\left(\gamma_{u i}\right)=a_{0}\left(a_{1}+\sqrt{b-\log \left(\gamma_{u i}\right)}\right) \\
& \rho_{2}\left(\gamma_{u i}\right)=a_{0}\left(a_{1}-\sqrt{b-\log \left(\gamma_{u i}\right)}\right),
\end{aligned}
$$

where,

$$
\begin{aligned}
& a_{0}=\frac{\sqrt{2 \delta_{1}^{2} \delta_{0}^{2}\left(\delta_{1}^{2}-\delta_{0}^{2}\right)}}{\delta_{1}^{2}-\delta_{0}^{2}} \\
& a_{1}=\frac{\delta_{1}^{2} \mu_{0}-\delta_{0}^{2} \mu_{1}}{\sqrt{2 \delta_{1}^{2} \delta_{0}^{2}\left(\delta_{1}^{2}-\delta_{0}^{2}\right)}} \\
& b=\frac{\left(\mu_{1}-\mu_{0}\right)^{2}}{2\left(\delta_{1}^{2}-\delta_{0}^{2}\right)}+\log \left(\sqrt{\frac{\delta_{1}^{2}}{\delta_{0}^{2}}}\right) .
\end{aligned}
$$

Following similar assumptions of the channels, $G\left(\gamma_{v i}\right)$ is expressed as,

$$
G\left(\gamma_{v i}\right)=E_{1}\left(\gamma_{v i}\right)
$$

where $E_{1}$ is the exponential integral function given by $E_{1}(x)=$ $\int_{x}^{\infty} \frac{e^{-t}}{t} d t$. Then, (14) can be simplified as,

$$
\gamma_{v i}=\gamma_{v i}=W\left(\frac{\lambda_{2 i}}{\lambda_{3 i}} \sigma_{i}\right)
$$

where $W$ is the Lambert function [13], which is the solution of $x \exp (x)=y$, i.e., $x=W(y)$.

Also, we find that $K_{01}\left(\gamma_{u i}\right)$ can be expressed as follows,

$$
K_{01}\left(\gamma_{u i}\right)=Q\left(f_{2}\left(\gamma_{u i}\right)\right)-Q\left(f_{1}\left(\gamma_{u i}\right)\right)
$$

where $\mathrm{Q}($.$) is the Q-function, the functions f_{1}($.$) and f_{2}($.$) are$ expressed as,

$$
\begin{aligned}
& f_{1}\left(\gamma_{u i}\right)=\frac{\rho_{1}\left(\gamma_{u i}\right)-\mu_{1}}{\delta_{1}} \\
& f_{2}\left(\gamma_{u i}\right)=\frac{\rho_{2}\left(\gamma_{u i}\right)-\mu_{1}}{\delta_{1}}
\end{aligned}
$$

Finally, the upper bound value of $\gamma_{u i}$ is found as,

$$
\gamma_{u i}^{(m)}=Q^{-1}\left(P_{D}\right) \delta_{1}+\mu_{1}
$$

\section{Simulation RESUlts}

In this section we evaluate the proposed scheme versus the benchmark scheme via numerical results. We consider the parameters mentioned in Table I to evaluate both the with sensing (proposed) and without sensing (benchmark) systems by checking the secondary minimum achievable EPG, and the outage metric, which is defined as the event where there is no feasible solution of the corresponding optimization problem.

TABLE I

SIMULATION PARAMETERS.

\begin{tabular}{|l|l|}
\hline Parameter Name & Value \\
\hline Sensing averaging bits $\left(N_{s}\right)$ & 1000 \\
\hline Maximum PT power $\left(P_{\max }\right)$ & $26.9897 \mathrm{dBm}$ \\
\hline Maximum ST power & $26.9897 \mathrm{dBm}$ \\
\hline \# Monte-Carlo iterations & 10000 \\
\hline Wireless channels & Rayleigh, \\
& Slow Flat Fading \\
\hline \# Subcarriers $(N)$ & 8 \\
\hline$Q_{\text {int }}$ & $-6 \mathrm{dBm}$ \\
\hline Minimum Rate $\left(R_{\min }\right)$ & 0.1 \\
\hline
\end{tabular}

It is known that the minimum EPG is resulted from minimizing the secondary transmission power and the corresponding rate. However, note that we have a minimum rate constraint $R_{\text {min }}$. Therefore, we define the EPG outage probability metric, which is the probability of outage that happens because there is no feasible solution that satisfies all the problem's constraints, as follows,

$$
\text { EPG Outage }= \begin{cases}0 & ; \text { A feasible solution exists } \\ 1 & ; \text { No feasible solution exists }\end{cases}
$$

Figure 2(a) shows the numerical results of both the proposed (with sensing) scheme and the benchmark (no sensing) scheme. The proposed scheme outperforms the benchmark scheme with at least 7 $\mathrm{dBm}$ for a similar EPG outage. It also shows the improvement of the EPG outage probability versus the increment of $Q_{i n t}$ for both the proposed and benchmark schemes. 
(a)

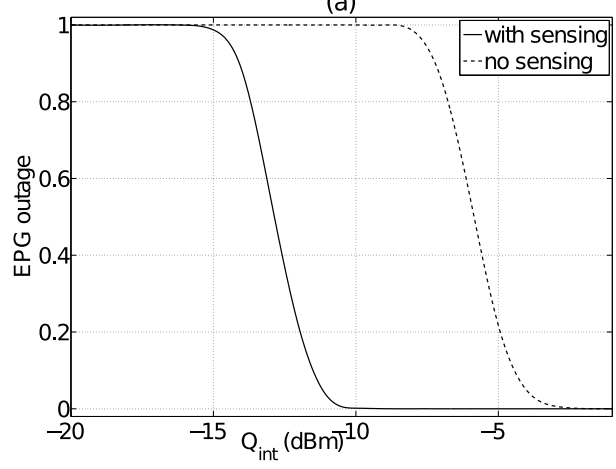

(d)

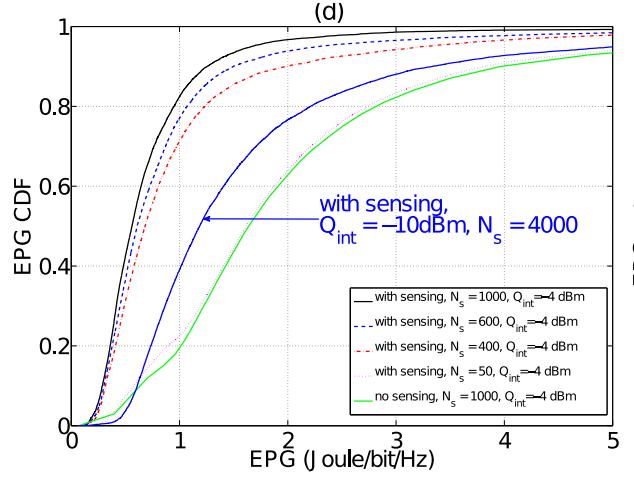

(b)

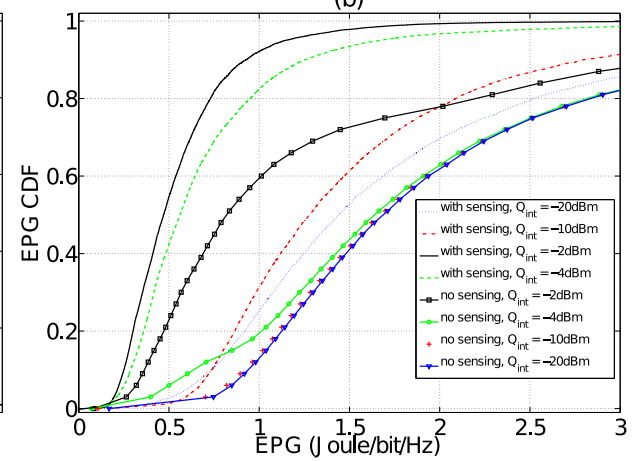

(e)

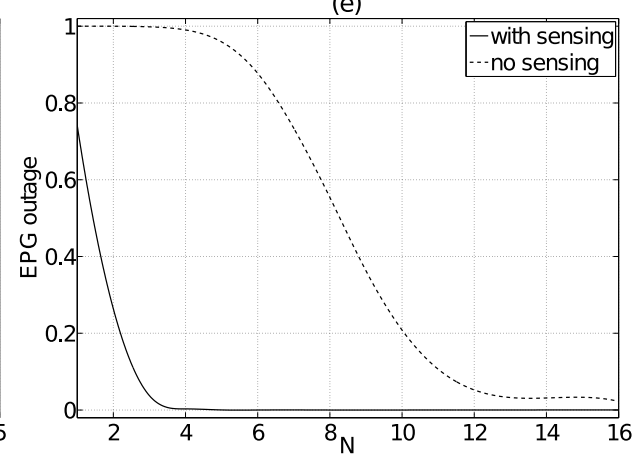

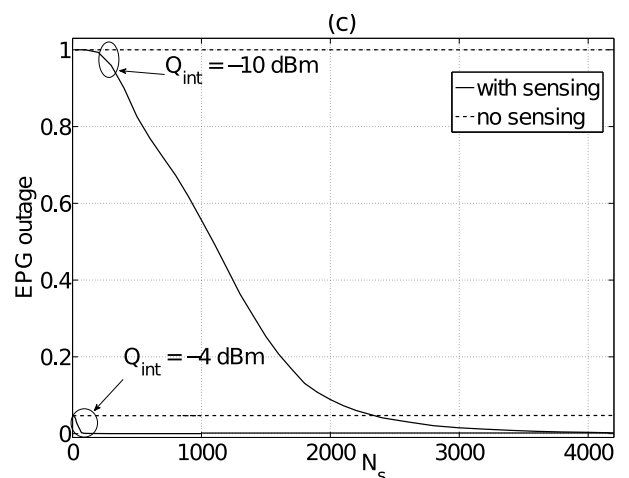

(f)

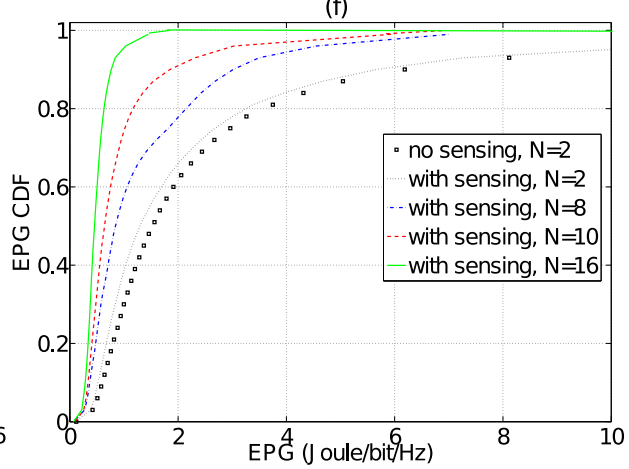

Fig. 2. Numerical results: EPG Outage versus (a) $Q_{\text {int }}$ (c) $N_{s}$ with $Q_{\text {int }}=-10$ and $-4 \mathrm{dBm}$ (e) $N$ with $Q_{\text {int }}=-10$ and $-4 \mathrm{dBm}$, for all schemes. EPG CDF for different (b) $Q_{\text {int }}$ (d) $N_{s}$ and $Q_{\text {int }}=-4 \mathrm{dBm}$ (f) $N$ for all schemes.

Figure 2(b) shows the CDF performance of the EPG metric for both the proposed and the benchmark schemes for several values of $Q_{\text {int }}$. The proposed scheme outperforms the benchmark scheme with minimum of 0.2 EGP units up-to around 1.5 EPG units for several values of $Q_{i n t}=-20,-10,-4,-2$. It is observed that the EPG performance of both the proposed and benchmark schemes degrades by decreasing the $Q_{\text {int }}$ values. We also note that the lower the values of $Q_{\text {int }}$ the lower the margin degradation of EPG performance of both the proposed and benchmark schemes, e.g. the performance degradation of the benchmark scheme from $Q_{\text {int }}=-2$ $\mathrm{dBm}$ to $Q_{\text {int }}=-4 \mathrm{dBm}$ is about 0.9 EPG units, while the degradation of the benchmark scheme from $Q_{i n t}=-10 \mathrm{dBm}$ to $Q_{i n t}=-20 \mathrm{dBm}$ is not observable.

Figure 2(c) depicts the EPG outage performance of both the proposed and benchmark schemes versus $N_{s}$ for $Q_{i n t}=-10 \mathrm{dBm}$ and $-4 \mathrm{dBm}$. As we know from Figure 2(a) the performance improves by increasing the threshold $Q_{\text {int }}$ which is consistent with the results of Figure 2(c). In Figure 2(c) we note the improvement of the proposed scheme performance by increasing the number of sensing samples, while for the benchmark scheme the performance does not change by changing $N_{s}$. It is observed that the margin of improvement of the proposed scheme depends on other parameters, such as $Q_{i n t}$. For $Q_{i n t}=-4 \mathrm{dBm}$ we notice an improvement of about 0.045 EPG outage probability, while for $Q_{\text {int }}=-10 \mathrm{dBm}$ we notice higher performance improvement, reaches about 1 EPG outage probability.

Figure 2(d) depicts the CDF performance of the EPG metric for both the proposed and benchmark schemes with $Q_{i n t}=-4 \mathrm{dBm}$ and different values of $N_{s}$. It is observed that EPG performance of the proposed scheme improves by increasing the value of $N_{s}$. The margin of this improvement begin to decrease for high values of $N_{s} \approx 1000$. We note that decreasing $N_{s}$ to very small values $(\approx<50)$ degrades the performance of the proposed scheme to a performance as worse as the benchmark scheme. It is important to note that increasing $N_{s}$ does not continuously improve the performance, but only to a certain value. This is inferred from the plot of $Q_{\text {int }}=-10 \mathrm{dBm}, N_{s}=4000$ of Figure 2(d) comparing to $Q_{\text {int }}=-4 \mathrm{dBm} N_{s}=1000$ of Figure 2(d), where the performance of the later one outperforms the formal one because $Q_{\text {int }}$ of the later one is higher, even though the $N_{s}$ of the later is lower.

Figure 2(e) shows the EPG outage performance of both proposed and benchmark schemes versus $N$ (sub-carriers number). We note that by increasing $N$ the EPG outage performance improves for both the proposed and benchmark schemes. The improvement is faster for the case of the proposed scheme comparing to the benchmark scheme.

Figure 2(f) shows the CDF performance of the EPG metric for both the proposed and benchmark schemes with different values of $N=2,8,10$, and 16. It is observed that the CDF performance of the EPG metric improves by increasing $N$ for the proposed scheme.

\section{CONCLUSION}

In this paper we proposed a hybrid system with a mixture of energy efficiency and cognitive radio resource allocation. The proposed allocation scheme utilizes the soft sensing information obtained from the secondary user of the energy detector for the primary user signal. The original proposed problem is not clearly convex, but we convert it to a convex problem to guarantee the unique solution. We evaluated and analyzed the proposed scheme with respect to the same system model without utilizing the soft sensing information (benchmark schem). The numerical results show that the proposed scheme outperforms the benchmark scheme with about $7 \mathrm{dBm}$. It is also shown that increasing the sub-carriers number from 2 to 16 improves the EPGperformance of the proposed scheme up-to 6 EPG 
units.

\section{APPENDIX}

In this section we prove the feasibility of the problem $P_{1}$, to guarantee the existence of a solution. Since the proof of convexity of the objective function is done in [8], we tackle the convexity of all inequality constraints.

Since it is straightforward to prove the convexity of constraints (4b) and (4c), we discuss the convexity of constraint (18). It is easy to infer that (18) is convex with respect to both $\gamma_{s i}$ (represented as linear function of $\gamma_{s i}$ ) and $\gamma_{v i}$ (represented as exponential integral function of $\gamma_{v i}$, which is also convex). However, because we have difference of two Q-function as a function of $\gamma_{u i}$, it is a little bit tricky to prove the convexity of this part of the constraint. The convexity of this part of the constraint depends on the parameters of the problem.

- If $f_{1}\left(\gamma_{u i}\right)$ and $f_{2}\left(\gamma_{u i}\right)$ have different signs, then the constraint is convex (concave), so it is still feasible.

- If $f_{1}\left(\gamma_{u i}\right)$ and $f_{2}\left(\gamma_{u i}\right)$ have the same sign, then we are not sure that we have convex (concave) constraint over all $\gamma_{u i}$. This case results in difference of two convex functions.

We use a convexification of a monotonic problem [14] approach to prove the convexity of the constraint [15]. [14] proved that it is possible to change a monotonic problem into a convex (concave) problem, which is enough to prove feasibility of the problem. It is easy to prove that (18) is monotonic in-term of $\gamma_{u i}$ (also in-term of all optimization variables). From the definitions of $\rho_{1}, \rho_{2}, f_{1}$ and $f_{2}$ at $(25,26$ and 30) we notice that:

1) $\rho_{1}$ is a decreasing function in terms of $\gamma_{u i}$, and so is $f_{1}\left(\gamma_{u i}\right)$.

2) $\rho_{2}$ is an increasing function in terms of $\gamma_{u i}$, and so is $f_{2}\left(\gamma_{u i}\right)$.

Now, we prove monotonicity of the difference of two Q-functions, $h\left(\gamma_{u i}\right)=Q\left(f_{2}\left(\gamma_{u i}\right)\right)-Q\left(f_{1}\left(\gamma_{u i}\right)\right)$, by taking derivative with respect to $\gamma_{u i}$ as follow

$$
\frac{\partial h}{\partial \gamma_{u i}}=\underbrace{\frac{\partial Q}{\partial f_{2}\left(\gamma_{u i}\right)}}_{\ominus} \underbrace{\frac{\partial f_{2}\left(\gamma_{u i}\right)}{\partial \gamma_{u i}}}_{\oplus}-\underbrace{\frac{\partial Q}{\partial f_{1}\left(\gamma_{u i}\right)}}_{\ominus} \underbrace{\frac{\partial f_{1}\left(\gamma_{u i}\right)}{\partial \gamma_{u i}}}_{\ominus}
$$

where, $\ominus$ represent a negative sign of the derivative (decreasing function), and $\oplus$ represent a positive sign of the derivative (increasing function). This proves that the overall $h\left(\gamma_{u i}\right)$ is a monotonic decreasing function in term of $\gamma_{u i}$.

From [14], we can convexificate (concavificate) a monotonically decreasing (increasing) functions, means we change the monotonic function into a convex (concave) function. Let $h$ be a twice differentiable function on X. For any $y=\left(y_{1}, \ldots, y_{n}\right)^{T} \in \Re_{+}^{n}$, define a $y^{1 / p}$ as the vector $\left(y_{1}^{1 / p}, \ldots, y_{n}^{1 / p}\right)^{T}$. We define a $p^{t h}$ power convexification transformation of $h$ as follows:

$$
\Psi_{h}(y)=\left[h\left(y^{1 / p}\right)\right]^{p} .
$$

where, $h(x)=Q\left(f_{2}(x)\right)-Q\left(f_{1}(x)\right)$, and $x$ is replaced by $\gamma_{u i}$.

Theorem A.1. Assume that there exist two positive numbers, $\epsilon_{0}>0$ and $\epsilon_{1}>0$, such that

$$
\begin{aligned}
& h(x) \geq \epsilon_{0}, \forall x \in X, \\
& \frac{\partial h}{\partial x_{j}} \leq-\epsilon_{1}, \forall x \in X \text { and } \forall j \in 1, \ldots, n .
\end{aligned}
$$

Then there exists a $p_{1}>0$ such that $\Psi_{h}(y)$ is a convex function of $y$ on $Y_{p}$, where,

$$
Y_{p}=y \in \Re^{n} \mid l_{j}^{p} \leq y_{j} \leq u_{j}^{p}, j=1, \ldots, n
$$

Applying the above theorem (A.1) on Ineq. (18), we can change it from a monotonically decreasing function into a convex function as follows:

$$
\sigma_{s i} E_{1}\left(\gamma_{v i}\right) \Psi_{h}(y) \leq Q_{i n t}^{\prime p},
$$

\section{REFERENCES}

[1] C. Han, T. Harrold, and etc., "Green radio: radio techniques to enable energy-efficient wireless networks," Communications Magazine, IEEE, vol. 49, no. 6, pp. $46-54$, june 2011.

[2] Z. Hasan, H. Boostanimehr, and V. Bhargava, "Green cellular networks: A survey, some research issues and challenges," Communications Surveys Tutorials, IEEE, vol. 13, no. 4, pp. 524 -540, quarter 2011.

[3] D. Feng, C. Jiang, G. Lim, J. Cimini, L.J., G. Feng, and G. Li, "A survey of energy-efficient wireless communications," IEEE Communications Surveys Tutorials, vol. 15, no. 1, pp. 167-178, 2013.

[4] G. Li, Z. Xu, C. Xiong, C. Yang, S. Zhang, Y. Chen, and S. Xu, "Energyefficient wireless communications: tutorial, survey, and open issues," IEEE Wireless Communications, vol. 18, no. 6, pp. 28-35, 2011.

[5] I. F. Akyildiz, W.-Y. Lee, M. C. Vuran, and S. Mohanty, "Next generation/dynamic spectrum access/cognitive radio wireless networks: A survey," Computer Networks, vol. 50, no. 13, pp. 2127 - 2159, 2006.

[6] V. Asghari and S. Aissa, "Resource sharing in cognitive radio systems: Outage capacity and power allocation under soft sensing," in Global Telecommunications Conference, 2008. IEEE GLOBECOM 2008. IEEE, 30 2008-dec. 4 2008, pp. 1 -5.

[7] R. Fischer and J. Huber, "A new loading algorithm for discrete multitone transmission," in Global Telecommunications Conference, 1996. GLOBECOM '96., vol. 1, nov 1996, pp. $724-728$ vol.1.

[8] R. S. Prabhu and B. Daneshrad, "An energy-efficient water-filling algorithm for OFDM systems," in IEEE International Conference on Communications (ICC), 2010, 2010, pp. 1-5.

[9] H. Volos and R. Buehrer, "Cognitive engine design for link adaptation: An application to multi-antenna systems," IEEE Transactions on Wireless Communications, vol. 9, no. 9, pp. 2902 -2913, september 2010.

[10] S. Srinivasa and S. Jafar, "Soft sensing and optimal power control for cognitive radio," IEEE Transactions on Wireless Communications, vol. 9 , no. 12 , pp. $3638-3649$, december 2010.

[11] F. F. Digham, M. Alouini, and M. K. Simon, "On the energy detection of unknown signals over fading channels," IEEE Transactions on Communications, vol. 55, no. 1, pp. 21-24, Jan. 2007.

[12] S. Boyd and L. Vandenberghe, Convex Optimization, 2004.

[13] R. M. Corless, G. H. Gonnet, D. E. G. Hare, D. J. Jeffrey, and D. E. Knuth, "On the Lambert-W function," in Advances in Computational Mathematics, 1996, pp. 329-359.

[14] D. Li, X. Sun, M. Biswal, and F. Gao, "Convexification, concavification and monotonization in global optimization," Annals of Operations Research, vol. 105, pp. 213-226, 2001.

[15] M.-S. Cheon, "Global optimization of monotonic programs: Applications in polynomial and stochastic programming." Georgia Institute of Technology, 042005. 